INPLASY

PROTOCOL

To cite: Zhou et al.

Arthroscopic Treatment for

Femoroacetabular

Impingement Syndrome in

Adolescents: A Systematic

Review and Meta-analysis.

Inplasy protocol 202180007.

doi:

10.37766/inplasy2021.8.0007

Received: 02 August 2021

Published: 02 August 2021

Corresponding author:

Xiang Zhou

1810122424@pku.edu.cn

Author Affiliation:

Peking University.

Support: None.

Review Stage at time of this submission: Piloting of the study selection process.

Conflicts of interest:

None declared.

\section{Arthroscopic Treatment for} Femoroacetabular Impingement Syndrome in Adolescents: A Systematic Review and Meta-analysis

Zhou, X1; Huang, ZG².

Review question / Objective: The aim of this meta-analysis is to evaluate the outcomes after hip arthroscopic treatment performed for FAI in adolescents and its complications and the revision rate.

Condition being studied: Femoroacetabular impingement syndrome (FAl) is one of the most common types of hip injury in teenagers at present, and it is also the precursor of a series of lesions such as osteoarthritis. Continuous hip pain and limited sports ability have severe interference to the daily activities of teenagers. At present the main treatment for FAl is hip arthroscopy therapy. Its advantages include few postoperative complications and high patient satisfaction as demonstrated in previous studies. However, there has been no consensus about FAl therapy specifically in adolescents. Most adolescents (younger than 21 years) have skeletal immaturity, which is characterized by an unclosed epiphyseal line. For this group, we need to consider whether normal surgery is still applicable, whether it will affect the epiphysis, whether it will affect the future development of femurs and also the way to prevent cam regrowth.

INPLASY registration number: This protocol was registered with the International Platform of Registered Systematic Review and Meta-Analysis Protocols (INPLASY) on 02 August 2021 and was last updated on 02 August 2021 (registration number INPLASY202180007).

\section{INTRODUCTION}

Review question / Objective: The aim of this meta-analysis is to evaluate the outcomes after hip arthroscopic treatment performed for FAl in adolescents and its complications and the revision rate.
Condition being studied: Femoroacetabular impingement syndrome (FAl) is one of the most common types of hip injury in teenagers at present, and it is also the precursor of a series of lesions such as osteoarthritis. Continuous hip pain and limited sports ability have severe 
interference to the daily activities of teenagers. At present the main treatment for FAl is hip arthroscopy therapy. Its advantages include few postoperative complications and high patient satisfaction as demonstrated in previous studies. However, there has been no consensus about FAl therapy specifically in adolescents. Most adolescents (younger than 21 years) have skeletal immaturity, which is characterized by an unclosed epiphyseal line. For this group, we need to consider whether normal surgery is still applicable, whether it will affect the epiphysis, whether it will affect the future development of femurs and also the way to prevent cam regrowth.

\section{METHODS}

Participant or population: Adolescents (patient age $<21$ years) with Femoroacetabular impingement syndrome (both cam-type and pincer-type) will be included. Patients have FAl combined with other disease (slipped capital femoral epiphysis, Legg-Calve-Perthes disease) will be excluded.

Intervention: The main intervention is hip arthroscopy treatment. All kinds of surgeries will be included, including Acetabuloplasty, osteochondroplasty, Femoroplasty, Labral debridement, Labral repair, etc. Open surgery and physical excercises will be excluded.

Comparator: Comparsions are made between patients' preoperative and postoperative status. The preoperative status is set as an control, all adolescent patients with untreated FAl will be included. Patients underwent revision surgery will be excluded.

Study designs to be included: There is no restrictions on the types of study. Case series and cohort study are common types of study in this field, and both types will be included.

Eligibility criteria: To be included in this study, the publications must focus on the primary arthroscopic treatment for FAI in adolescents (patient age $<21$ years). Patients have FAl combined with other disease (slipped capital femoral epiphysis, Legg-Calve-Perthes disease) were excluded. Articles involved comparison between adults and adolescents were excluded. Only English language studies, formal articles were included. Conference abstract, expert commentary, systematic review, meta-analysis and articles with no quantity analysis were excluded.

Information sources: The information will be acquired from five electronic databases including PubMed, Scopus, Cochrane Library, EMBASE, MEDLINE.

Main outcome(s): The main outcomes include the postsurgical modified Harris Hip Score (mHHS), the Hip Outcome Score (HOS)-Activities of Daily Living (ADL), the HOS-Sports-Specific Subscale (SSS), the Non-Arthritic Hip Score (NAHS), the visual analog scale (VAS) for pain, the revision rate, the alpha-angle and satisfaction.

Quality assessment / Risk of bias analysis: Two authors will independently assess. Any disagreement will be resolved by discussion until consensus is reached or by consulting a third author to decrease bias. An instrument for the assessment of case series studies developed by Yang et al will be used. Four factors will be considered: (1) Study aims and design, (2) Descriptions of treatment protocol, (3) Descriptions of methods and therapeutic/side-effects, (4) Conduct of the study.

Strategy of data synthesis: The WMD will be calculated for the main outcomes including the modified Harris Hip Score (mHHS), the Hip Outcome Score (HOS)Activities of Daily Living (ADL), the HOSSports-Specific Subscale (SSS). The minimum number of studies needed for calculation is three. Meta-analysis will be conducted using the random-effects model to pool WMD. 'Metafor' package in $R$ will be used.

Subgroup analysis: In adolescents, the incidence of cam deformity is very prominent, and studies have shown that 
the movement before and after epiphyseal closure is closely related to the occurrence and development of cam deformity. So the patients with cam-type FAI (combined with pincer-type FAl will also be included) will be included in a subgroup to compare with the total FAI population.

Sensitivity analysis: A subgroup analysis for cam-type FAl is one part of sensitivity analysis in our study. And further sensitivity analysis will only be conducted if heterogeneity is high enough (I^2>25\%).

Country(ies) involved: China.

Keywords: Femoroacetabular Impingement; Adolescent; Arthroscopy; Cam deformity.

Contributions of each author:

Author 1 - Xiang Zhou.

Author 2 - Zhi-Gao Huang. 